\title{
Oxygen-mediated Polymerization Initiated by Oltipraz-derived Thiones
}

\author{
Scott R. Zavada, ${ }^{a}$ Joseph C. Furgal, ${ }^{b}$ Nathan D. Wood ${ }^{b}$ and Timothy F. Scott ${ }^{a, b}$
}

${ }^{a}$ Macromolecular Science and Engineering Program, University of Michigan, Ann Arbor, MI 48109-2136, USA

${ }^{b}$ Department of Chemical Engineering, University of Michigan, Ann Arbor, MI 48109-2136, USA

Correspondence to: Timothy F. Scott (E-mail: tfscott@umich.edu)

((Additional Supporting Information may be found in the online version of this article.))

\section{ABSTRACT}

A pyrrolopyrazine-thione derived from oltipraz, a compound that has been investigated as a chemopreventive agent, affords radicals in the presence of thiols and oxygen via a redox cycle, an attribute that suggests its suitability as an initiator for oxygen-mediated polymerization. Here, we explore the utilization of this pyrrolopyrazine-thione, generated in situ from a precursor, as an initiator for the radical-mediated thiol-ene polymerization. While the pyrrolopyrazine-thione was shown to be capable of generating radicals in the presence of atmospheric oxygen and thiol groups, the reaction extents achievable were lower than desired owing to the presence of unwanted side reactions that would quench radical production and, subsequently, suppress polymerization. Moreover, we found that complex interactions between the pyrrolopyrazinethione, its precursor, oxygen, and thiol groups determine whether or not the quenching reaction dominates over those favorable to polymerization.

\section{INTRODUCTION}

Numerous applications, including paints, coatings, and adhesives, rely on in situ polymerization, where a liquid monomer formulation is transformed, via a polymerization reaction, into a solid polymer in the location where the material is utilized. This transformation is effected by exposing the liquid monomer formulation to some initiation stimulus, most commonly light, ${ }^{2}$ elevated temperature, ${ }^{3}$ or a chemical reactant. ${ }^{4}$ Often, the chemical stimulus, frequently labelled as a 'cross-linker', 'curing agent', or 'hardener', is packaged separately from the primary liquid monomer resin and the polymerization reaction only proceeds upon mixing of the reaction components. These two-component systems, examples of which include epoxy adhesives ${ }^{5}$ and urethane coatings, ${ }^{6}$ usually require precise mixing of the two reactants as deviating from the ideal ratio could lead to incomplete cross-linking, hindering the development of optimal properties. Alternatively, instead of storing the reactants in separate packages, the polymerization can rely upon a compound that is naturally and reliably present in the environment where the resultant polymer will be utilized. The development of suitable systems that respond to such environmentally-borne initiation stimuli could permit rapid advances in several emerging technologies, notably self-healing materials and surgical adhesives.

The list of environmentally-borne, chemical initiation stimuli primarily includes components of the atmosphere. Of these, nitrogen ${ }^{7}$ and $\operatorname{argon}^{8}$ can be readily dismissed owing to their low reactivity; carbon dioxide can similarly be discarded for being relatively unreactive ${ }^{9}$ as well as its typically low atmospheric concentration. This leaves water ${ }^{10-12}$ and oxygen, ${ }^{13-15}$ both of which have been employed as polymerization initiation stimuli. The utilization of atmospheric moisture is

This is the author manuscript accepted for publication and has undergone full peer review but has not been through the copyediting, typesetting, pagination and proofreading process, which may lead to differences between this version and the Version record. Please cite this article as doi:10.1002/ pola.28507. 
especially well known for initiating the polymerization of alkyl cyanoacrylates. In the presence of moisture or any weak nucleophile, alkyl cyanoacrylates undergo anionic polymerization, yielding poly(alkyl cyanoacrylates). ${ }^{10}$ Of these, shorter chain (e.g., methyl and ethyl) alkyl cyanoacrylates are utilized for commonly-available consumer glues, while the longer alkyl chain (e.g., octyl) variants serve as surgical-grade adhesives owing to their hydrolysis resistance and thus improved biocompatibility. ${ }^{16,} 17$ In addition to alkyl cyanoacrylates, moisture-cured isocyanatebased coatings ${ }^{12}$ and silicone-based sealants ${ }^{11}$ utilize atmospheric water to effect polymerization. Nevertheless, while moisture-cured adhesives and coatings certainly play an important role in many commercial products, there are inherent drawbacks that preclude their broader adoption in new applications. Notably, the reaction rates are strongly dependent upon the relative humidity, with polymerization nearly ceasing at very low concentrations of atmospheric moisture. ${ }^{18,19}$ Moreover, there are serious limitations as to the types of chemistries that can be utilized in a moisture-curing system: any system that reacts with water may also react with many weakly nucleophilic moieties, severely

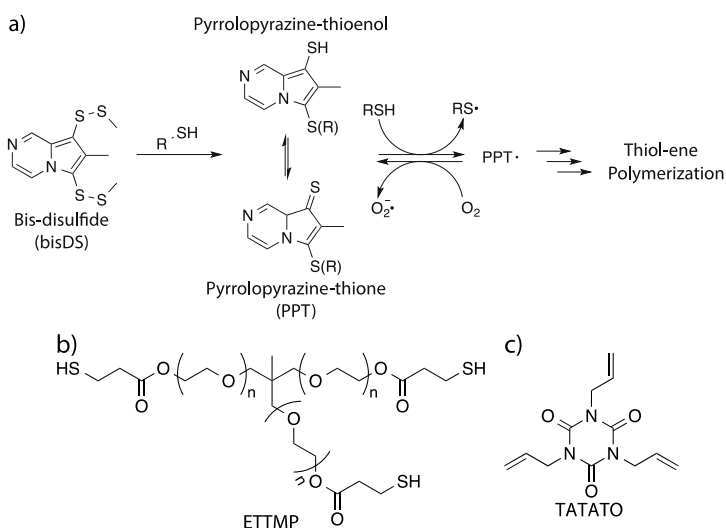

Scheme 1: Structures of the oxygen-mediated initiator and monomers used. a) The bisdisulfide (bisDS) derivative of oltipraz is converted to a PPT intermediate by reaction with a thiol. Subsequently, in the presence of both thiol and oxygen, the PPT participates in a radical-generating redox cycle, ${ }^{1}$ resulting in thiol-ene polymerization. b) Ethoxylated trimethylolpropane tri(3-mercaptopropionate) (ETTMP). c) 1,3,5-Triallyl-1,3,5-triazine2,4,6(1H,3H,5H)-trione (TATATO). limiting the type of functional groups that can be present. Thus, in searching for a broadly applicable environmentally-borne initiation stimulus, we are left with atmospheric oxygen, a particularly promising candidate given its relatively high atmospheric concentration and its reactivity.

Oxygen has long served as the initiator for oil-based paints and coatings, where unsaturated fatty acids are cross-linked through sluggish oxygen-mediated reactions, catalysed by metal driers; oilbased paints typically do not fully cure until weeks or months after application. ${ }^{20} \mathrm{~A}$ more generally useful free-radical polymerization mechanism is one based on the radicalmediated chain-growth polymerization of vinyl monomers, notably acrylates and methacrylates, ${ }^{21}$ whereby formulations of monomers and a latent initiating species polymerize rapidly by exposing the initiator to its radical-generating stimulus. Although oxygen is well-suited for generating polymerization-initiating radicals, owing to its capacity for participating in redox reactions, ${ }^{22}$ radical-mediated chain-growth polymerizations are typically strongly inhibited by oxygen. ${ }^{23}$

The radical-mediated thiol-ene addition reaction, where a thiol and an electron-rich double bond form a thioether linkage, proceeds in the presence of radicals. ${ }^{24,25}$ In contrast to conventional radical-mediated chain-growth polymerizations, the ready hydrogen abstractability from the ubiquitous thiol functionalities in thiol-ene formulations affords a mechanism that is extraordinarily resistant to molecular oxygen at atmospheric concentrations. Notably, the utilization of oxygen to initiate thiol-ene polymerization has already been established. One such system utilized oxidoreductase enzymes as the basis for initiating systems capable of converting aqueous thiol-ene monomer formulations into hydrogels upon exposure to the atmosphere. $^{15}$ Another system employed alkylboranes, species capable of rapidly generating free radicals in the presence of oxygen. ${ }^{26}$ While both of these systems have utility, there are limitations: enzymes may lack stability in non-aqueous systems, while the extreme reactivity of alkylboranes may 
also lead to long-term stability problems. Given these limitations, there remains a need to develop alternative oxygenmediated polymerization approaches.

Interestingly, one such alternative approach arises from cancer research. 1,2Dithiole-3-thiones, a class of compounds to which the cancer chemopreventive drug oltipraz belongs, have been shown to induce cellular production of chemoprotective phase II detoxification enzymes. ${ }^{27}$ The major metabolite of oltipraz is a pyrrolopyrazinethione, which is produced in the presence of glutathione, an intracellular tripeptide thiol. This pyrrolopyrazine metabolite is relatively stable in the absence of oxygen; however, in the presence of further thiol and oxygen, thiyl and peroxy radicals are formed. Moreover, the metabolite can be conveniently liberated in situ via the action of thiol on 7-methyl-6,8bis(methyldisulfanyl) pyrrolo[1,2-a]pyrazine (for brevity, referred to as the 'bis-disulfide' or 'bisDS'), a disulfide precursor (see Scheme 1a). ${ }^{28}$ Thus, an approach utilizing such compounds appears particularly wellsuited to the oxygen-mediated initiation of a thiol-ene polymerization. Whereas a thiolene resin formulated with a disulfide precursor in an inert atmosphere would form the pyrrolopyrazine-thione intermediate in situ, exposure to oxygen would subsequently generate radicals capable of initiating the polymerization. Here we investigate the suitability of bisDS as an oxygen-mediated initiator for thiolene polymerization.

\section{EXPERIMENTAL}

\section{Materials}

Ethoxylated trimethylolpropane tri(3mercaptopropionate) (ETTMP, $1300 \mathrm{~g} / \mathrm{mol}$ ) was obtained from Evans Chemetics. Poly(ethylene glycol (PEG, $600 \mathrm{~g} / \mathrm{mol}$ ), dimethyl sulfoxide (DMSO), 1,3,5- triallyl-1,3,5-triazine2,4,6(1H,3H,5H)-trione (TATATO), sodium thiomethoxide, methyl methane thiol sulfonate (MMTS), methyl-3-propionate and 5,5dimethyl-1-pyrroline $\mathrm{N}$-oxide (DMPO) were obtained from Sigma-Aldrich. Oltipraz was obtained from LKT Laboratories. All materials were used as received. Thiol-ene resins were formulated from $83.6 \mathrm{wt} \%$ ETTMP and $16.4 \mathrm{wt} \%$ TATATO such that the thiol to allyl stoichiometric ratio prior to polymerization was maintained at 1:1 for all experiments.

The synthesis of bisDS followed a previously published procedure. ${ }^{28}$ Briefly, $1.22 \mathrm{~g}$ of oltipraz was dissolved in $200 \mathrm{~mL}$ of ethanol under nitrogen and heated to $44^{\circ} \mathrm{C}$. To this solution, $1.89 \mathrm{~g}$ of sodium thiomethoxide was added while stirring. After 45 minutes, $3.5 \mathrm{~mL}$ of MMTS was added and stirred for 2 hours. The solvent was removed under vacuum and the resulting crude product was dissolved in $\mathbf{4 0}$ $\mathrm{mL}$ of chloroform and filtered. Upon sitting, the filtrate phase separated and the clear, oily layer was discarded. The solvent was again removed under vacuum and the resulting product placed in a freezer overnight, affording crystals. The crystals were washed with cold ethanol and recrystallized from hot ethanol. ${ }^{1} \mathrm{H}$ NMR $\left(400 \mathrm{MHz}, \mathrm{CDCl}_{3}\right), \delta: 2.48(\mathrm{~d}, 6 \mathrm{H}), 2.57$ (s, $3 \mathrm{H}), 7.83(\mathrm{~d}, 1 \mathrm{H}), 8.24(\mathrm{dd}, 1 \mathrm{H}), 9.08(\mathrm{~d}, 1 \mathrm{H})$.

For experiments performed under anaerobic conditions, monomers and solvents were subjected to at least six freeze-pump-thaw cycles to ensure rigorous deoxygenation prior to use, and formulations were assembled and mixed in an anaerobic chamber. For the aerobic experiments, formulations were assembled under atmospheric conditions using airsaturated monomers.

\section{UV-Vis spectroscopy}

Ultraviolet-visible (UV-Vis) spectroscopy was performed using an Agilent Technologies Cary 60 UV-Vis spectrophotometer. Formulations were placed in a $1 \mathrm{~mm}$ pathlength quartz cuvette immediately after mixing, whereupon spectra from 300 to 700 $\mathrm{nm}$ were collected every 10 minutes for 5 hours.

\section{EPR spectroscopy}

Electron paramagnetic resonance (EPR) spectroscopy was performed using a Bruker EMX spectrometer. A TM 110 cavity (ER 4103TM, Bruker), $100 \mathrm{kHz}$ modulation 


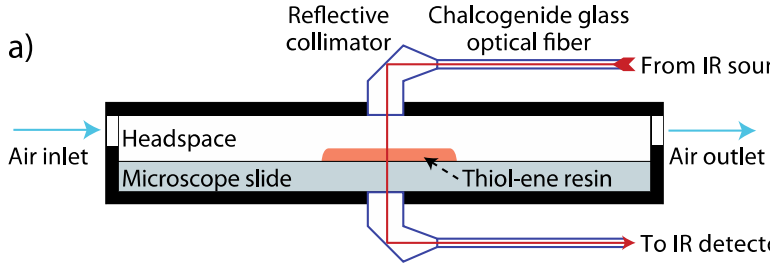

b)

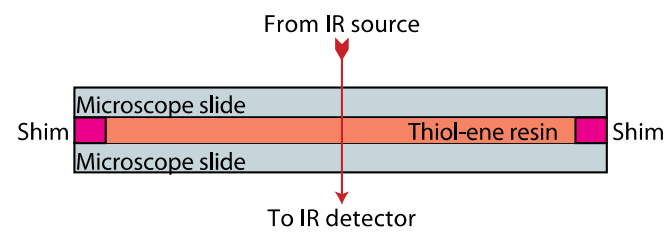

c)

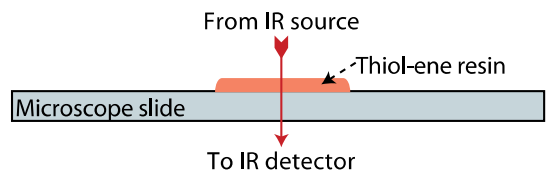

Scheme 2: Schematic diagrams of experimental configurations used to examine oxygenmediated polymerization kinetics by FTIR spectroscopy. a) A sealed sample cell permits measurement of IR spectra under anaerobic conditions until air is introduced into the cell via inlet and outlet ports. b) Sandwiching a resin formulation, mixed under oxygen-saturated conditions, between two glass slides throughout IR spectra collection prevents additional oxygen from diffusing into the sample during polymerization. c) A resin formulation, mixed under oxygen-saturated conditions, is exposed to the atmosphere throughout IR spectra collection, permitting oxygen to diffuse into the sample during polymerization.

frequency, and $1 G_{p p}$ modulation amplitude were used. Sample formulations were placed in a flat quartz cell and all experiments were performed at room temperature using DMPO as a spin trap. For experiments performed under initially anaerobic experiments, deoxygenated bisDS, ETTMP, and DMPO were dissolved in DMSO, $600 \mu \mathrm{L}$ of which were injected into the flat quartz cell, and a spectrum was collected after two hours. Each formulation was then removed from the flat quartz cell, exposed to the atmosphere for three minutes, then reinjected back into the flat quartz cell, and a spectrum was collected after 15 minutes of oxygen exposure. For experiments performed under completely aerobic conditions, the air-saturated formulation components were combined and a spectrum was collected after 15 minutes.

\section{FTIR spectroscopy}

For experiments performed under initially anaerobic experiments, an approximately 20 $\mu \mathrm{m}$ thick film of deoxygenated resin was spread on a glass slide in a sealed sample cell. ${ }^{26}$ Infrared spectra of the resin formulations were monitored remotely and in transmission using a Nicolet 6700 Fourier transform infrared (FT-IR) spectrometer equipped with a fiber optic coupling accessory via chalcogenide optical fiber patch cables fitted with silver reflective collimators (see Scheme 2a). Air was introduced into the sample chamber via ports on either side, and the reaction was monitored by observing the disappearance of the allyl ether $\left(3100 \mathrm{~cm}^{-1}\right)$ and thiol $(2570$ $\mathrm{cm}^{-1}$ ) absorbance peaks, ${ }^{29}$ using the methyl peak at $4370 \mathrm{~cm}^{-1}$ as an internal standard. ${ }^{30}$,

${ }^{31}$ Spectra were collected at a rate of two per second and 64 spectra were averaged for every reported data point.

For experiments performed under aerobic conditions, air-saturated resin samples were either injected between two glass microscope slides separated by $50 \mu \mathrm{m}$ thick shims to prevent further atmospheric oxygen from diffusing into the sample (see Scheme 2b) or spread as an approximately $20 \mu \mathrm{m}$ thick film exposed to the atmosphere on a glass slide (see Scheme 2c). Each sample was placed in a Nicolet 6700 FT-IR spectrometer equipped with a horizontal transmission accessory and spectra were collected from 6400 to $2000 \mathrm{~cm}^{-1}$ at a rate of two per second, and the reaction was again monitored as described above.

\section{Rheometry}

Rheological measurements were performed using a TA Instruments ARES rheometer, configured with a $25 \mathrm{~mm}$ diameter parallelplate fixture. Sample formulations were combined and mixed under aerobic conditions and, immediately after $0.300 \mathrm{~mL}$ were deposited on the lower plate and the upper fixture was lowered to a gap of 0.5 $\mathrm{mm}$, data were collected at a rate of one point every 8 seconds using an oscillatory strain of $1 \%$ at a frequency of $1 \mathrm{~Hz}$. 


\section{NMR spectroscopy}

All ${ }^{1} \mathrm{H},{ }^{13} \mathrm{C}$ and heteronuclear single quantum coherence (HSQC) 2D NMR spectroscopy were performed in DMSO- $_{6}$ on a Varian $700 \mathrm{MHz}$ spectrometer. ${ }^{1} \mathrm{H}-\mathrm{NMR}$ spectra were collected at $700 \mathrm{MHz}$ using a 7998.4 $\mathrm{Hz}$ spectral width, a relaxation delay of $1 \mathrm{~s}$, a pulse width of $45^{\circ}$ and $65 \mathrm{k}$ data points. ${ }^{13} \mathrm{C}$ NMR spectra were collected at $176 \mathrm{MHz}$ using a $25000 \mathrm{~Hz}$ spectral width, a relaxation delay of $1.5 \mathrm{~s}, 75 \mathrm{k}$ data points and a pulse width of $40^{\circ}$.

\section{Mass Spectrometry}

Electrospray ionization mass spectrometry (ESI-MS) was performed on an Agilent Q-ToF Time-of-Flight mass spectrometer with ESI in positive ionization mode. Samples were prepared by adding $\sim 0.2 \mathrm{mg}$ analyte to $2 \mathrm{~mL}$ of solvent (acetonitrile). $10 \mu \mathrm{L}$ of solvent was injected into the instrument and analyzed for exact mass.

\section{RESULTS AND DISCUSSION}

Prior to the polymerization reaction that is anticipated to proceed upon exposure of a thiol-ene resin formulated with bisDS to atmospheric oxygen, a pyrrolopyrazinethione (PPT) reactive intermediate (in equilibrium with its thioenol form) must be generated in situ from the thiol-disulfide exchange reaction of bisDS with the thiol monomer (see Scheme 1). UV-Vis spectroscopy has previously been employed to monitor the PPT product formation from the reaction of bisDS with the monothiol glutathione; ${ }^{28}$ generation of the PPT is readily observed as its thiocarbonyl moiety strongly absorbs at approximately $300 \mathrm{~nm}$ and in the $420-500 \mathrm{~nm}$ range owing to the $\pi$ to $\pi^{*}$ and the forbidden $\mathrm{n}$ to $\pi^{*}$ transitions, respectively. ${ }^{32}$ In their investigation of bisDS in an aqueous solution, Fishbein and coworkers found that the absorbance peak at $450 \mathrm{~nm}$ reached its maximum value within one minute and they concluded that the PPT is rapidly generated in near quantitative yield. $^{28}$ UV-Vis experiments were performed here to determine whether the thiol functional groups affixed to the trithiol monomer ETTMP are similarly capable of generating the PPT in situ from bisDS. As the PPT participates in subsequent reactions in the presence of molecular oxygen, preliminary studies were performed under anaerobic conditions. Upon mixing of bisDS into a model thiol-ene resin composed of ETTMP and TATATO, an absorbance peak in the visible region of the spectrum $\left(\lambda_{\max }=500 \mathrm{~nm}\right)$ was observed to increase over 2 hours for all concentrations of bisDS examined (see Figure 1a-1c), indicating the generation of thiocarbonyl moieties and, hence, the PPT intermediate; as expected, the absorbance at $\lambda_{\max }$ increases linearly with raised bisDS concentration. Assuming complete conversion of bisDS into the $\mathrm{PPT},{ }^{28}$ the molar absorptivity, $\varepsilon$, was found to be 5930 $\pm 180 \mathrm{M}^{-1} \mathrm{~cm}^{-1}$. In contrast, when thiol groups were omitted, there was no observable change in the UV-Vis spectrum of a bisDS-incorporating solution of TATATO and PEG over five hours and no absorbance observed at $500 \mathrm{~nm}$ (see Figure 1d), confirming that thiol functional groups must be present for reaction with bisDS to afford the PPT reactive intermediate.

Furthermore, a 2D NMR and ESI mass spectral study of a model PPT system containing a 1:1 mixture of bisDS and a monofunctional thiol (methyl-3mercaptopropionate) was explored to verify the possible reactive intermediate structures. Although many intermediates were observed by ESI-MS (Table S1), these studies confirmed the formation of a monothione intermediate with the opposite disulfide functionalized with the methyl-3mercaptopropionate as the dominant species (Figures S1 and S2). The intermediates likely exist in a tautomeric equilibrium of thione and thioenol derivatives (Scheme 1a) as suggested by the formation of thione and disulfide-bridged structures observed in mass spectral analysis (Table S1). Residual bisDS starting material was not observed after the addition of the monofunctional thiol, demonstrating the reactive preference for the newly introduced R-thiol. 
a)

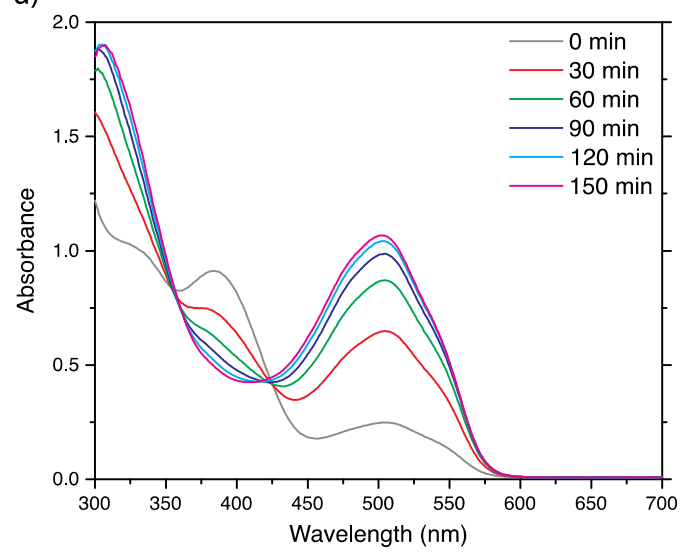

c)

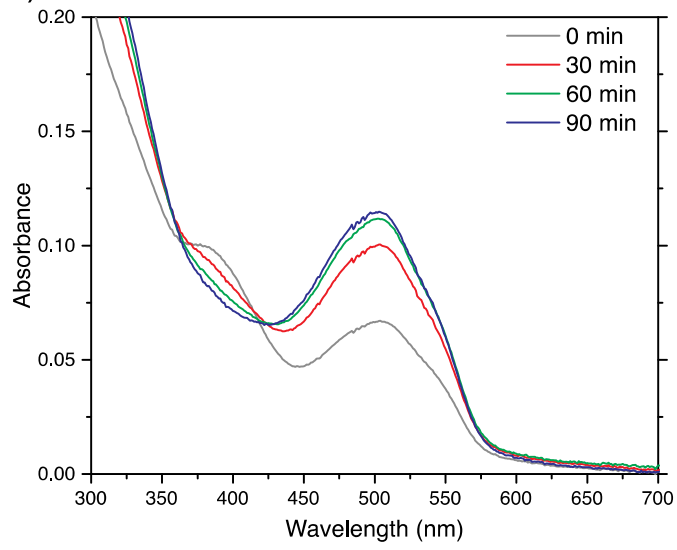

b)

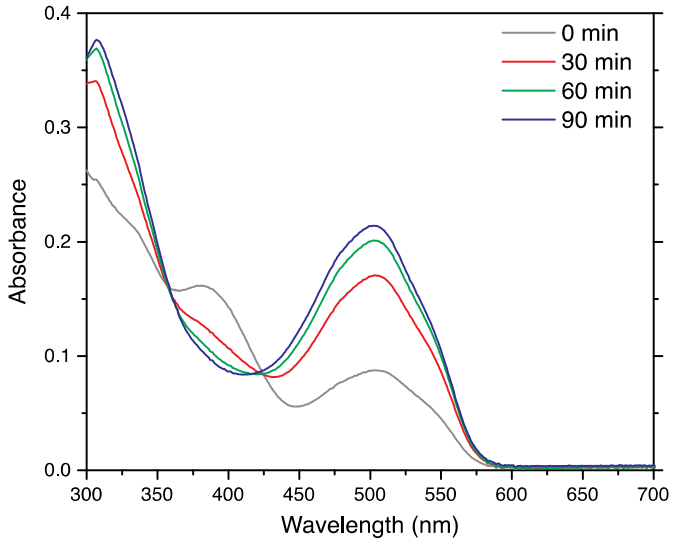

d)

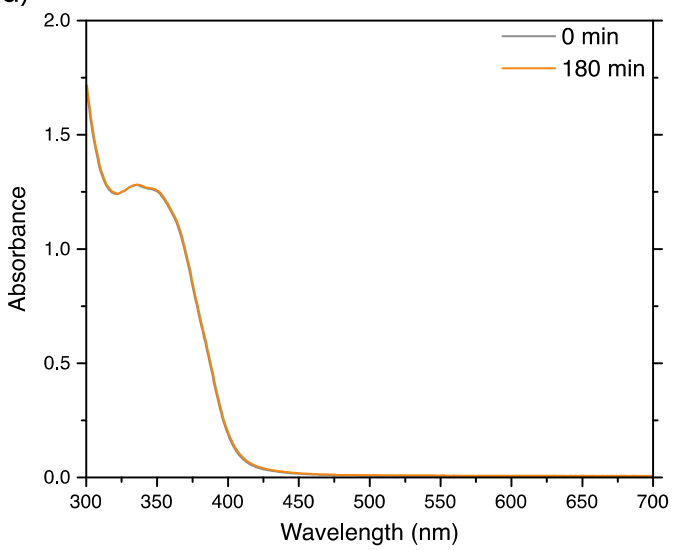

Figure 1: UV-Vis absorption spectra for bisDS-containing resin formulations mixed and maintained under anaerobic conditions. a) 0.05 wt\% bisDS in ETTMP and TATATO; b) 0.01 wt\% bisDS in ETTMP and TATATO; c) 0.005 wt\% bisDS in ETTMP and TATATO; and d) 0.05 wt\% in 83.6 wt\% PEG (i.e., thiol-free) and 16.4 wt\% TATATO.

Having established that the PPT intermediate is yielded by the reaction of bisDS and thiols, its oxygen-mediated radical generating characteristics were investigated using EPR spectroscopy by employing DMPO as a spin trap. In the absence of oxygen, no radicals were detected in a solution of 172 $\mu \mathrm{M}$ bisDS (equal to $0.005 \mathrm{wt} \%), 100$ equivalents of thiol functional groups, and an excess of DMPO in DMSO; a ten-fold increase in the bisDS concentration yielded the same negative result (see Figure S3), confirming that the reaction between bisDS and thiol to afford the PPT intermediate is not radical-mediated. However, upon exposure of the thiol/bisDS solutions to the atmosphere, radical generation was clearly evident in their EPR spectra (see Figure 2); an ETTMP formulation with 0.005 wt\% bisDS, 100 equivalents of thiol groups, and excess DMPO exhibited a strong signal consistent with those previously reported where superoxide and thiyl radicals were identified. ${ }^{1}$ Interestingly, when the bisDS concentration was increased from 0.005 wt\% to 0.05 wt\%, the resultant EPR signal intensity, and hence radical concentration, slightly decreased (Figure 2). The decomposition rates of conventional radical initiators are typically first order with respect to the initiator concentration, ${ }^{33}$ even for systems requiring a coinitiator, ${ }^{34}$ such that the number of radicals generated scales with the initiator concentration; however, this expected behaviour is not observed in the current system. Rather, at sufficiently high initiator (i.e., either bisDS or thiol) concentrations, increasing the amount of initiator in the monomer formulation actually reduces the number of radicals generated. This phenomenon may be explained by the mechanism proposed by 


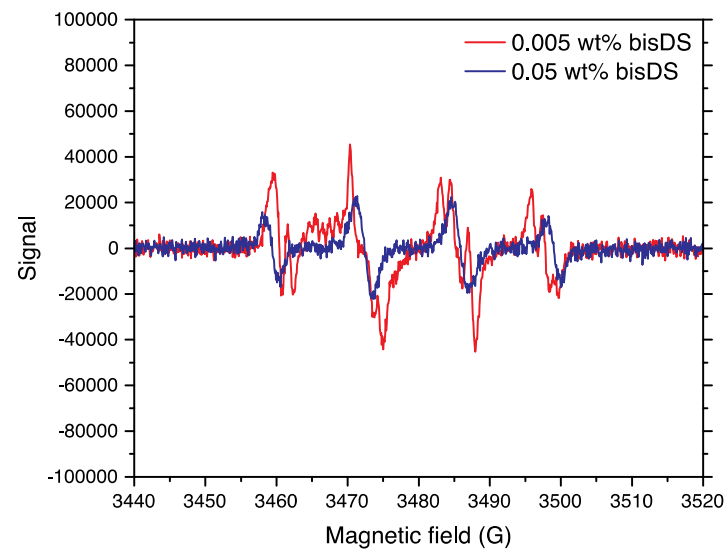

Figure 2: EPR spectra for solutions mixed under anaerobic conditions of bisDS, ETTMP, and DMPO in DMSO, collected 15 minutes after

Fishbein and coworkers, where an undesired quenching reaction, ${ }^{1}$ favoured at high PPT concentrations, shuts down the radicalgenerating redox cycle. Nevertheless, despite this counterintuitive behaviour, radical generation does proceed upon exposure of the PPT intermediate to both oxygen and thiol, demonstrating similar behaviour for bisDS in both aqueous environments $^{1}$ and in bulk thiol-ene monomer formulations examined here. This in turn suggests that bisDS could function as an oxygen-mediated initiator for thiol-ene polymerizations, although the maximum attainable polymerization rate may be limited by the reduced radical generation at raised bisDS or thiol concentrations.

a)

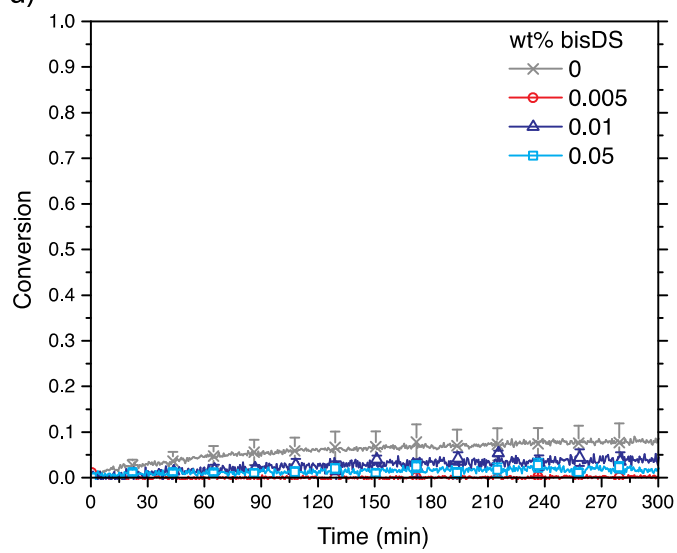

To evaluate this hypothesis, the polymerization of model thiol-ene resins, composed of ETTMP and TATATO and formulated with various concentrations of bisDS, was examined using FTIR spectroscopy to monitor the consumption of thiol and allyl functional groups during the reaction (Scheme 2a). In the absence of both oxygen and bisDS, the functional group conversion reached approximately $7 \%$ after five hours (see Figure $3 a$ and Figure S2a), attributable to the often poor stability of thiol-ene formulations which have been observed to slowly react even in the absence of an initiator. ${ }^{35}$ Although incorporation of an appropriate inhibitor can improve stability, ${ }^{36}$ inhibitors were not added to these formulations to avoid masking reactions caused by the bisDS. Notably, the formulations incorporating bisDS exhibited minimal functional group conversion in the absence of oxygen over five hours for all bisDS concentrations examined (Figure $3 a$ and Figure S2a), indicating that bisDS does not generate polymerization-inducing radicals itself and supporting the results observed in the EPR experiments discussed above (Figure 2). Indeed, no inhibitor was needed in the formulations as the PPT generated in situ appears to induce an inhibitory effect. b)

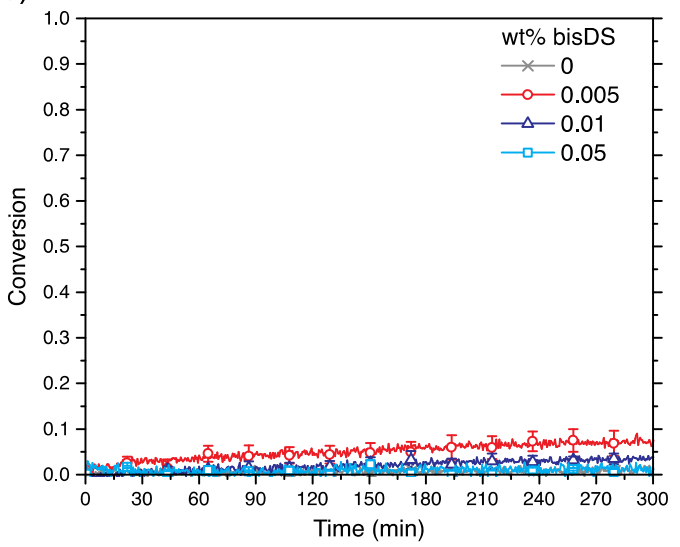

Figure 3: Thiol functional group conversions for bisDS-containing ETTMP/TATATO thiol-ene formulations mixed under anaerobic conditions. Experiments performed by a) maintaining anaerobic conditions, or b) exposing the samples to the atmosphere. 
Having established the minimal reaction progression under anaerobic conditions, these resin formulations were investigated for their behaviour upon exposure to atmospheric oxygen. As the UV-Vis experiments demonstrated that 2-3 hours were required to fully generate the PPT (see Figure 1), formulations were prepared under anaerobic conditions and left undisturbed for 3 hours prior to exposing them to atmospheric oxygen (see Figure $3 b$ and Figure S2b). Upon exposure to the atmosphere, the observed polymerization rates were low, with functional groups conversions for the resin incorporating 0.005 wt\% bisDS, the fastest polymerizing formulation of those examined, reaching only $7 \%$ after 5 hours exposure. Notably, although radical generation was demonstrated in formulations containing bisDS and thiol functionalities upon exposure to atmospheric oxygen using EPR spectroscopy, this did not translate into significant oxygen-mediated thiol-ene polymerization. Moreover, these polymerization kinetics results support those observed by EPR spectroscopy where increasing the bisDS concentration beyond a particular value affords a decreased radical concentration, behaviour that is consistent with the hypothesis discussed above of a radical quenching reaction that inhibits polymerization in the presence of excess PPT. A mechanism that might account for the diminished numbers of radicals generated at raised PPT concentrations is proposed in Scheme 3. Here, the abstraction of a hydrogen from the thioenol tautomer of PPT affords a resonance-stabilized radical unable to participate in subsequent propagation or chain transfer reactions, leading to termination. This mechanism is analogous to that observed for conventional radical polymerization inhibitors such as monomethylether hydroquinone (MEHQ). ${ }^{37}$ Notably, MEHQ has previously been employed at low concentrations to retard a redox-initiated thiol-ene polymerization; ${ }^{4}$ however, in contrast to the current study, raised $\mathrm{MEHQ}$ concentrations resulted in enhanced thiol-ene polymerization rates.

In contrast to conventional, radicalmediated chain growth polymerization reactions, oxygen does not wholly inhibit

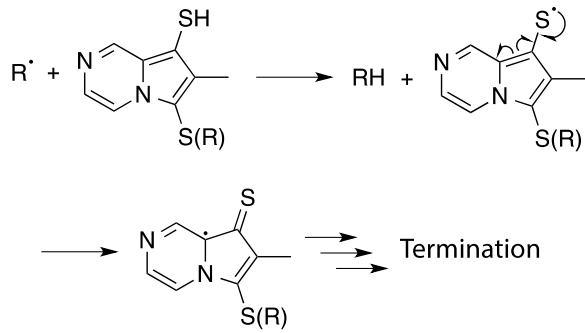

Scheme 3: Proposed polymerization inhibition mechanism. In the presence of excess PPT, an active radical abstracts a hydrogen from the thioenol tautomer, generating a resonancestabilized, unreactive PPT • intermediate, inhibiting the radical-affording redox cycle as well as removing PPT.

radical-mediated thiol-ene polymerizations; nevertheless, the presence of oxygen can retard polymerization rates even in thiolene systems. Thus, to prevent further oxygen from diffusing into the samples during polymerization, a second set of polymerization kinetics experiments was performed where oxygen-saturated ETTMP/TATATO resins were formulated with bisDS under atmospheric conditions, immediately injected between two glass microscope slides, and the consumption of thiol and allyl functional groups was monitored by FTIR spectroscopy (see Scheme 2b). In contrast to the polymerization kinetics observed for resins under constant atmospheric exposure, here the formulation incorporating 0.005 wt\% bisDS reached a reaction conversion of nearly $45 \%$ within 2 hours (Figure 4 and Figure S5). Unfortunately, as before, increasing the concentration of bisDS above 0.005 wt\% deceased both the rate and extent of polymerization, a dramatic divergence from previously studied thiolene systems where the scaling of the polymerization rate with the initiation rate approaches unity; ${ }^{29}$ indeed, the 0.05 wt\% bisDS formulation exhibiting a reaction extent after five hours that was actually lower than the formulation with no bisDS. Nevertheless, similar behaviour, where an increase in the initiator concentration results in a suppressed reaction rate, has been observed previously in other initiator systems for both radical-mediated acrylate ${ }^{38}$ and thiol-ene polymerization chemistries, ${ }^{15}$ including those that employ iron-based Fenton chemistry or camphorquinone/ 
tertiary amine photoinitiation systems at raised $\mathrm{Fe}^{2+}$ or amine concentrations, respectively. ${ }^{34}$ For such systems, the reaction rates decrease at elevated $\mathrm{Fe}^{2+}$ or amine concentrations owing to the occurrence of unwanted inhibitory and termination reactions. Similarly, the behavior exhibited here may be attributable to the aforementioned quenching reaction that suppresses radical generation at high PPT concentrations, accounting for the difference in behavior between the experiments seen in Figures 3 and 4. Under initially anaerobic conditions, bisDS is converted into the PPT intermediate over several hours, such that the PPT concentration is high when atmospheric oxygen is introduced. Conversely, when the formulation is initially oxygen-saturated, the PPT generated by reaction of bisDS with thiol can immediately react with further thiol and oxygen to start the radicalgenerating redox cycle; thus, as the concentration of PPT is initially low such that the quenching reaction is minimized, initiating radicals are readily formed and the polymerization is able to proceed. The monomer conversion plateaus for formulations with both 0.005 and 0.01 wt\% bisDS after approximately 2 hours reaction time suggest complete consumption of the dissolved oxygen such that radicalgenerating redox cycle ceases after that time.

Given the significant differences in polymerization kinetics between samples

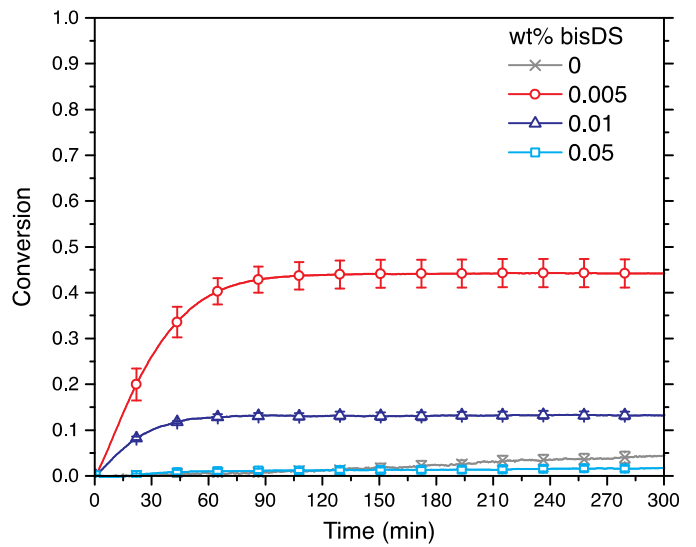

Figure 4: Thiol functional group conversions for bisDS-containing ETTMP/TATATO thiol-ene formulations mixed under oxygen-saturated conditions. Experiments performed by sandwiching samples between glass slides, preventing additional oxygen diffusion into each formulation. initially anaerobic but exposed to the atmosphere throughout their reaction and those initially oxygen-saturated but reacting within closed cells, additional UV-Vis and EPR experiments investigating the influence of oxygen on the PPT evolution were performed. Initially, oxygen-saturated thiolene resins were formulated with bisDS at various concentrations and UV-Vis spectra were collected on the resultant solutions over time. The results of the UV-Vis experiments run under either oxygensaturated or anaerobic conditions were superficially similar as the peaks at both 300 and $500 \mathrm{~nm}$ increased over time (see Figure 5); however, unlike the experiments performed under anaerobic conditions where the complete conversion of bisDS into the PPT intermediate took several hours (see Figure 1), the absorbance at $\lambda_{\max }$ did not plateau in the presence of oxygen even after 5 hours for any of the bisDS concentrations examined (Figure 5). Moreover, the observed $\lambda_{\max }$ absorbances did not scale linearly with the initial bisDS concentration; using the PPT molar absorptivity determined above, the conversion of bisDS to PPT after five hours for 0.005 wt $\%, 0.01$ wt $\%$, and 0.05 wt $\%$ bisDS were $29 \%, 65.1 \%$, and $83.3 \%$, respectively. Thus, when oxygen was initially present, the PPT generation was retarded and, at lower bisDS concentrations, the PPT concentration remained much lower than it did under anaerobic conditions, indicating that oxygen either inhibits the formation of the PPT from bisDS or it interacts with the PPT generated by the reaction between bisDS and thiol. To determine which of these mechanisms occurs, a formulation of 0.05 wt\% bisDS in ETTMP and TATATO was initially assembled under anaerobic conditions and left undisturbed for three hours to wholly convert bisDS to the PPT (see Figure 6). Subsequently, this formulation was exposed to atmospheric oxygen for 8 hours, whereupon the absorbance at $\lambda_{\max }$ had dropped considerably (Figure 6), indicating that oxygen does indeed react with the generated PPT. Interestingly, after five hours under anaerobic conditions, the $\lambda_{\max }$ absorbance partially recovered, indicating 
a)

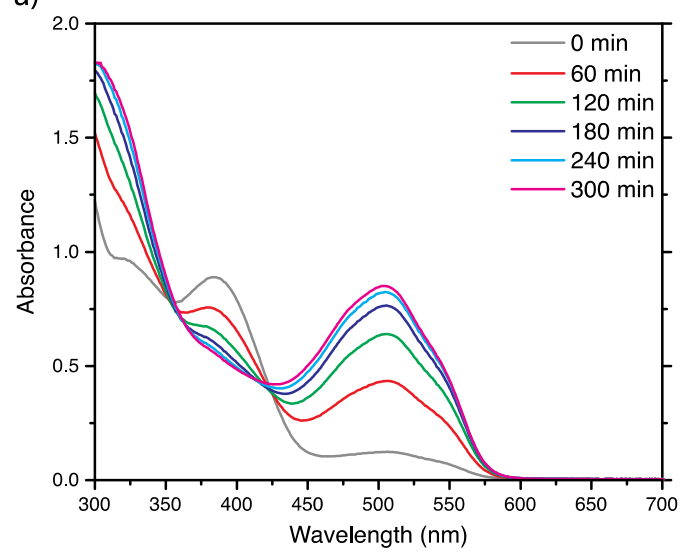

b)

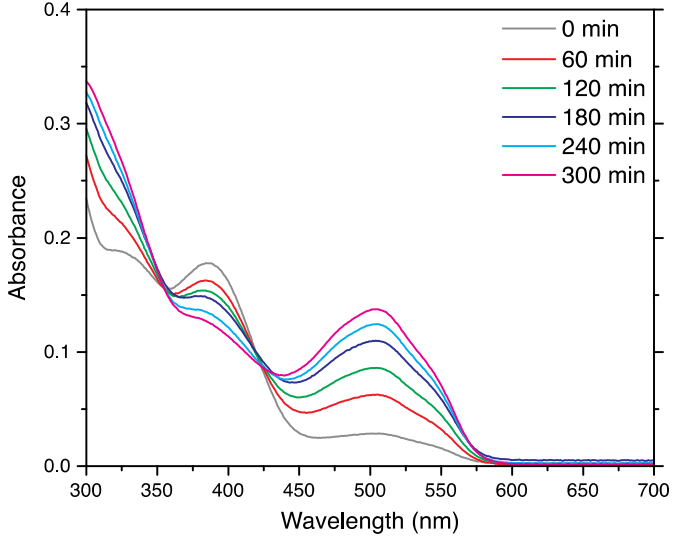

c)

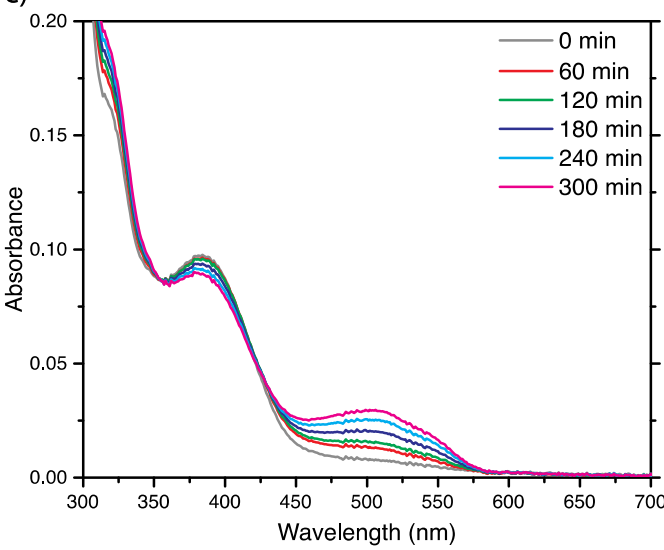

Figure 5: UV-Vis absorption spectra for bisDS-containing thiol-ene formulations mixed under oxygensaturated conditions. a) 0.05 wt\% bisDS in ETTMP and TATATO; b) 0.01 wt\% bisDS in ETTMP and TATATO; and c) 0.005 wt\% bisDS in ETTMP and TATATO.

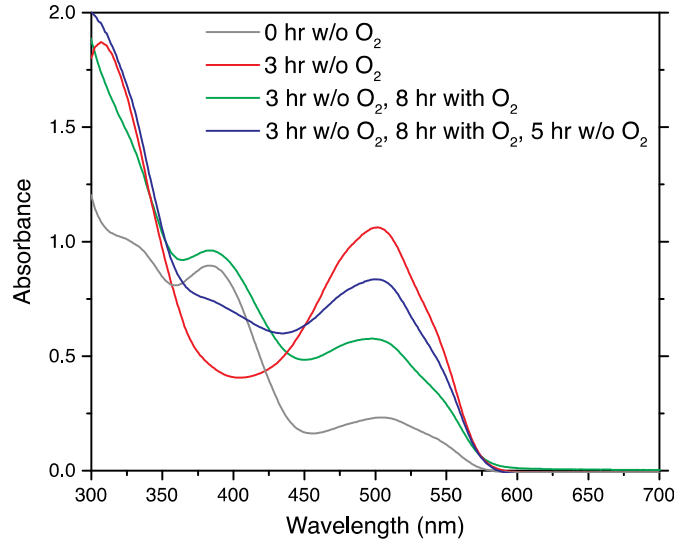

Figure 6: UV-Vis absorption spectra for a thiolene formulation of 0.05 wt\% bisDS in ETTMPTATATO immediately after mixing under anaerobic conditions, after 3 hours maintained under anaerobic conditions, after 8 hours of subsequent atmosphere exposure, and finally after 5 hours sealed in a cuvette to eliminate further oxygen diffusion.

that the interactions between bisDS, PPT, thiol, and oxygen are in part reversible.

Equivalent experiments examining the generation of radicals in oxygen-saturated solutions were performed using EPR spectroscopy. Here, a solution with 0.005 $w t \%$ bisDS and 100 equivalents of thiol exhibited a much stronger signal after reaction under initially aerobic conditions (see Figure 7) than when the experiment was performed under initially anaerobic conditions (Figure 2). This again is likely a result of the significantly lower PPT concentration, as observed in the UV-Vis experiments (Figure 5), when oxygen is initially present and supports the hypothesis that formulations with low PPT concentrations are more capable of generating free radicals via the redox cycle as the, polymerization-retarding influence of the quenching reaction dominates at higher PPT concentrations. 


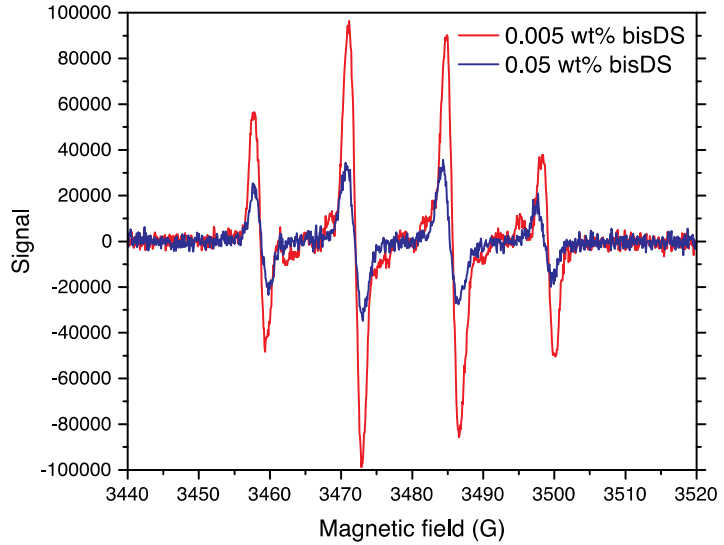

Figure 7: EPR spectra for solutions mixed under oxygen-saturated conditions of bisDS, ETTMP, and DMPO in DMSO, collected 15 minutes after mixing.

To further investigate the role of oxygen in the radical-generating behaviour of bisDS, additional polymerization kinetics experiments were performed under aerobic conditions (Scheme 2c): the resins, with bisDS concentrations ranging from 0 to 0.25 $w t \%$ were formulated in the presence of atmospheric oxygen and were continually exposed to the atmosphere throughout the reaction (see Figure $8 \mathrm{a}$ and Figure $\mathrm{S4a}$ ). At bisDS concentrations from 0 to 0.025 wt\%, the polymerization rate and extent increased, as the bisDS concentration was raised and the maximum rate occurred at the start of the reaction. The monomer conversion after five hours reaction time for the resin formulated with 0.05 wt\% bisDS

a)

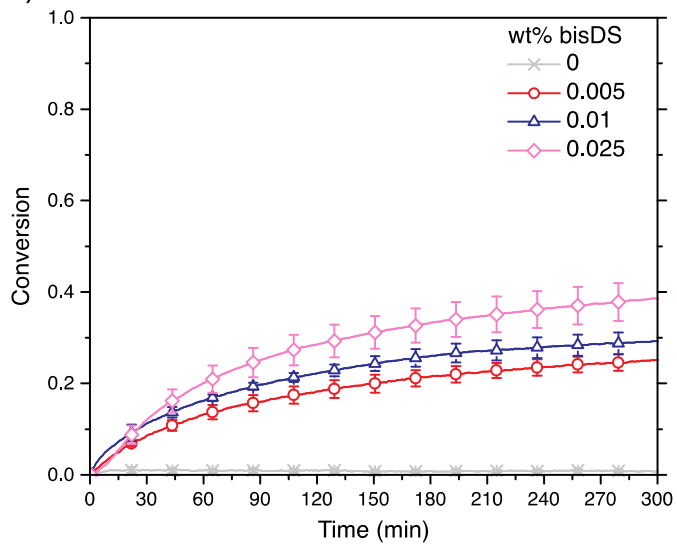

was similar to that for the 0.025 wt\% bisDS resin, although an induction period of approximately 20 minutes was apparent prior to the polymerization onset (Figure $8 \mathrm{~b}$ and Figure S4b). Notably, of the experimental conditions examined, only the conditions used here effected appreciable monomer conversion with 0.05 wt\% bisDS. Moreover, resin formulated with 0.075 wt\% bisDS achieved the the highest monomer conversion of any of the reaction conditions examined, where after 5 hours the thiol and allyl conversions were $51.8 \pm 4.6 \%$ and $49.9 \pm 3.5 \%$, respectively (Figure $8 b$ ), which increased to $60.8 \pm 4.2 \%$ and $57.8 \pm 3.5 \%$, respectively, after 8 hours. This again suggests that the role of oxygen is complex, participating both in generating radicals from the PPT and thiol while simultaneously eliminating the PPT via some quenching reaction. Whereas removing the PPT lessens the potential radical generation rate, it also reduces the occurrence of the polymerization-retarding quenching reaction. Thus, the constant influx of oxygen from the atmosphere destroyed excess PPT, suppressing the quenching reaction, while still retaining a sufficient PPT concentration to generate polymerization-initiating radicals. This hypothesis is further supported by the behavior at high bisDS concentrations where progressively longer induction times and decreased polymerization rates and extents were observed as bisDS concentrations were

b)

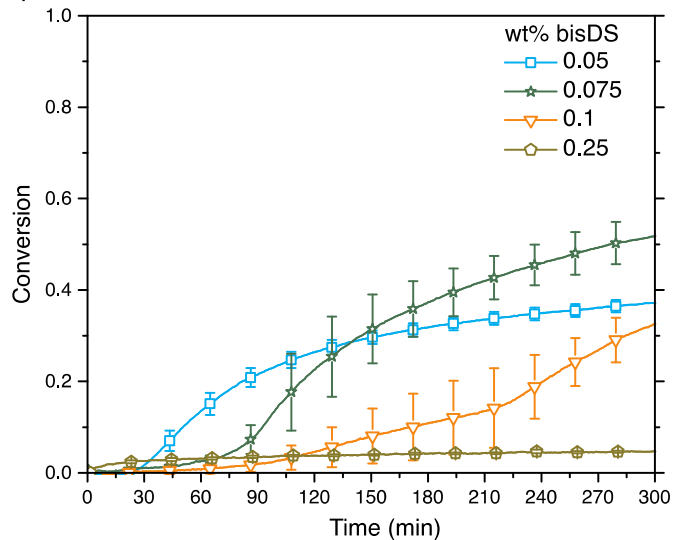

Figure 8: Thiol functional group conversions for bisDS-containing ETTMP/TATATO thiol-ene formulations mixed under oxygen-saturated conditions. Experiments performed by exposing the samples to the atmosphere. For clarity, bisDS-containing resin compositions separated into two ranges: a) $0-0.025$ wt\% bisDS; b) $0.05-0.25$ wt $\%$ bisDS. 
raised above 0.05 wt\% (see Figure $8 b$ and Figure S4b); indeed, at a concentration of 0.25 wt\% bisDS, the monomer conversion after five hours was only $5 \%$, appreciably lower than all bisDS-containing formulations examined and indicating that the PPT concentration generated by this bisDS loading was too high to enable sufficient radical generation for significant polymerization.

Finally, to confirm that the model, multifunctional thiol-ene resin in the presence of both bisDS and atmospheric oxygen results in the formation of crosslinked polymer, parallel plate rheometry was used to monitor oxygen-mediated polymerizations. The experimental conditions used here were similar to those used for the polymerization kinetics experiments performed using initially oxygen-saturated resins that were subsequently sealed throughout the polymerization reaction; however, whereas the formulations were made under aerobic conditions and most of the monomer/bisDS

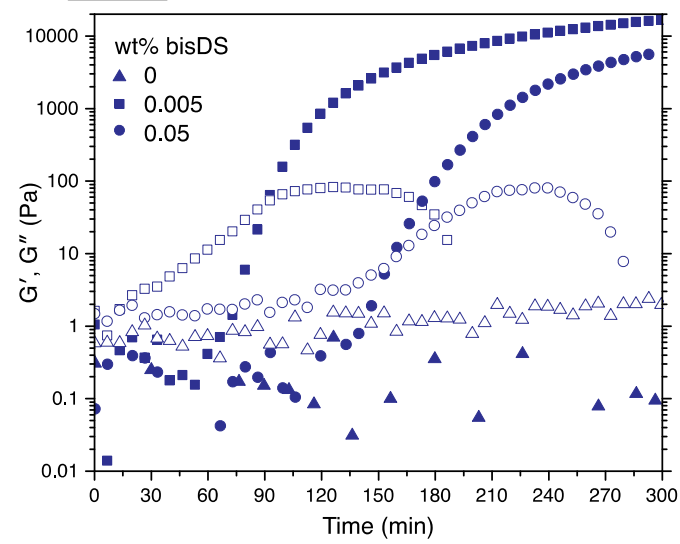

Figure 9: Storage ( $G^{\prime}$, closed symbols) and loss ( $G^{\prime \prime}$, open symbols) moduli, measured by parallel plate rheometry, of bisDS-containing ETTMP/TATATO thiol-ene formulations mixed under oxygen-saturated conditions.

formulation" was sealed against the atmosphere by the rheometer plates, diffusion of oxygen from the atmosphere still occurred at the plate edges (see Figure 9). A control resin formulated without bisDS displayed essentially no change in either storage or loss modulus and remained liquid over five hours, indicating negligible polymerization over that period. When bisDS was included in the formulation at either 0.005 wt\% or 0.05 wt\%, a cross-over of the storage and loss moduli, indicative of gelation, ${ }^{39}$ was observed. Whereas the cross-over point for the 0.005 wt\% bisDS formulation was reached after 91 minutes, the cross-over for the 0.05 wt\% formulation occurred significantly later at 158 minutes. Moreover, the $0.05 \mathrm{wt} \%$ formulation only achieved significant gelation in a ring around the edge, while the 0.005 wt\% bisDS formulation exhibited solid polymer throughout the sample, results consistent with those observed in the polymerization kinetics experiments.

\section{CONCLUSIONS}

Incorporation of bisDS in model thiol-ene resin formulations has been shown to afford a PPT intermediate product, attributable to a reaction between bisDS and the thiol functional groups in the resin. Exposure of these bisDS-incorporating thiol-ene resins to atmospheric oxygen results in the generation of radicals via a redox cycle, in the presence of both thiol and molecular oxygen, permitting its utilization as an oxygen-mediated initiating system for thiolene polymerization. An unwanted side reaction dominates at elevated PPT concentrations that quenches the redox cycle, preventing radical formation and retarding polymerization. Although the influence of this quenching unfortunately limits the utility of bisDS as an oxygenmediated initiator of thiol-ene polymerization, we anticipate that the development of approaches to suppress the quenching reaction will afford a rapid and efficient method of utilizing oxygen as an environmentally-borne polymerization initiation stimulus.

\section{ACKNOWLEDGEMENTS}

The authors gratefully acknowledge funding from the National Aeronautics and Space Administration (NASA Space Technology Research Fellowship, award number NNX12AM31H) and the National Institutes of Health (NIH R21, award number R21 EB011761). 


\section{REFERENCES AND NOTES}

1. M. Velayutham, F. A. Villamena, M. Navamal, J. C. Fishbein and J. L. Zweier, Chem. Res. Toxicol., 2005, 18, 970-975.

2. N. B. Cramer and C. N. Bowman, J. Polym. Sci. A Polym. Chem., 2001, 39, 3311-3319.

3. L. W. Hill and S. B. Lee, J. Coating. Technol., 1999, 71, 127-133.

4. M. A. Cole, K. C. Jankousky and C. N. Bowman, Polym. Chem., 2013, 4, 11671175.

5. Y. Deng and G. C. Martin, Macromolecules, 1994, 27, 5147-5153.

6. D. K. Chattopadhyay and K. V. S. N. Raju, Prog. Polym. Sci., 2007, 32, $352-$ 418.

7. R. Y. Igarashi and L. C. Seefeldt, Crit. Rev. Biochem. Mol. Biol., 2003, 38, 351384.

8. L. Khriachtchev, M. Rasanen and R. B. Gerber, Acc. Chem. Res., 2009, 42, 183191.

9. A. I. Cooper, J. Mater. Chem., 2000, 10, 207-234.

10. S. Thennarasu, N. Krishnamurti and K. L. Shantha, J. Adhes. Sci. Technol., 1989, 3, 237-260.

11. J. Comyn, F. de Buyl, N. E. Shephard and C. Subramaniam, Int. J. Adhes. Adhes., 2002, 22, 385-393.

12. D. K. Chattopadhyay, B. Sreedhar and K. V. S. N. Raju, Polymer, 2006, 47, 38143825.

13. C. Navarra, C. Goodwin, S. Burton, B. Danieli and S. Riva, J. Mol. Catal. B: Enzym., 2010, 65, 52-57.

14. B. J. Berron, L. M. Johnson, X. Ba, J. D. McCall, N. J. Alvey, K. S. Anseth and C. N. Bowman, Biotechnol. Bioeng., 2011, 108, 1521-1528.

15. S. R. Zavada, N. R. McHardy and T. F. Scott, J. Mater. Chem. B, 2014, 2, 25982605.

16. D. Wachter, A. Bruckel, M. Stein, M. F. Oertel, P. Christophis and D. K. Boker, Neurosurg. Rev., 2010, 33, 483-489.
17. A. J. Singer and H. C. Thode, Jr., Am. J. Surg., 2004, 187, 238-248.

18. L. A. Lewis, R. W. Smithwick, G. L. Devault, B. Bolinger and S. A. Lewis, J. Forensic. Sci., 2001, 46, 241-246.

19. P. J. Mankidy, R. B. Ramakrishnan and H. C. Foley, Chem. Commun., 2006, 1139-1141.

20. R. van Gorkum and E. Bouwman, Coord. Chem. Rev., 2005, 249, 1709-1728.

21. D. Colombani, Prog. Polym. Sci., 1997, 22, 1649-1720.

22. P. M. Wood, Biochem. J., 1988, 253, 287-289.

23. A. K. O'Brien and C. N. Bowman, Macromolecules, 2006, 39, 2501-2506.

24. C. E. Hoyle and C. N. Bowman, Angew. Chem. Int. Ed., 2010, 49, 1540-1573.

25. C. E. Hoyle, A. B. Lowe and C. N. Bowman, Chem. Soc. Rev., 2010, 39, 1355-1387.

26. S. R. Zavada, N. R. McHardy, K. L. Gordon and T. F. Scott, ACS Macro Lett., 2015, 4, 819-824.

27. M. L. Clapper, Pharmacol. Ther., 1998, 78, 17-27.

28. M. Navamal, C. McGrath, J. Stewart, P. Blans, F. Villamena, J. Zweier and J. C. Fishbein, J. Org. Chem., 2002, 67, 94069413.

29. T. F. Scott, C. J. Kloxin, R. B. Draughon and C. N. Bowman, Macromolecules, 2008, 41, 2987-2989.

30. F. Westad, A. Schmidt and M. Kermit, J. Near Infrared Spectrosc., 2008, 16, 265273.

31. P. Hourant, V. Baeten, M. T. Morales, M. Meurens and R. Aparicio, Appl. Spectrosc., 2000, 54, 1168-1174.

32. K. Skrabania, A. Miasnikova, A. M. Bivigou-Koumba, D. Zehm and A. Laschewsky, Polym. Chem., 2011, 2, 2074-2083.

33. K. E. J. Barrett, J. Appl. Polym. Sci., 1967, 11, 1617-\&.

34. W. D. Cook, Polymer, 1992, 33, 600609. 
35. H. H. Szmant, A. J. Mata, A. J. Namis and A. M. Panthananickal, Tetrahedron, 1976, 32, 2665-2680.

36. W. D. Cook, F. Chen, D. W. Pattison, P. Hopson and M. Beaujon, Polym. Int., 2007, 56, 1572-1579.

37. H. Becker and H. Vogel, Chem. Eng. Technol., 2006, 29, 1227- 1231.

38. L. M. Johnson, B. D. Fairbanks, K. S. Anseth and C. N. Bowman,
Biomacromolecules, 2009, 10, 31143121.

39. H. H. Winter and F. Chambon, J. Rheol., 1986, 30, 367-382.
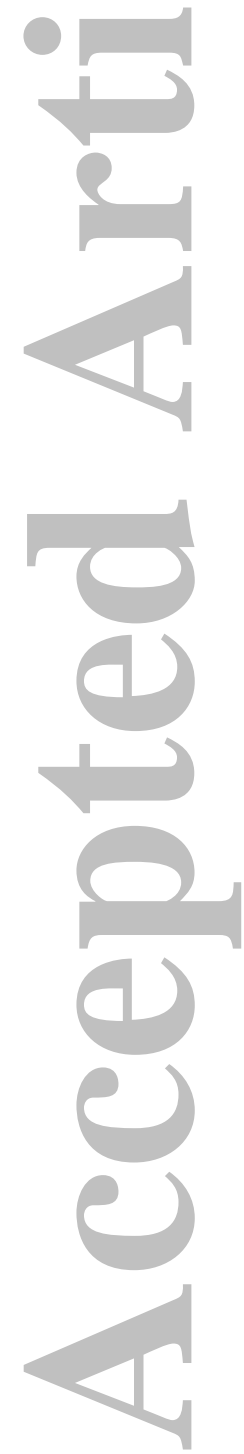